\title{
Grief After Perinatal Loss
}

A.T. Pereira' ${ }^{1}$ D. Mota ${ }^{2}$, F. Martins Alves 3 , C. da Costa Campos ${ }^{4}$, L. Ribeiro², J.D. Rodrigues²

'Vila Nova de Gaia/Espinho Healthcare Center Vila Nova de Gaia- Portugal, Department of Psychiatry and Mental Health, Vila Nova de Gaia, Portugal.

2Vila Nova de Gaia/Espinho Hospital Center, Psychiatry Department, Vila Nova de Gaia, Portugal.

3Unidade Local de Saúde do Alto Minho, Psychiatry Department, Viana do Castelo, Portugal.

${ }^{4}$ Hospital de Braga, Psychiatry Department, Braga, Portugal.

The loss of an infant through stillbirth, miscarriage, or neonatal death is recognized as a traumatic life event. In a populationbased sample, bereaved individuals who had lost a child showed the highest prevalence of complicated grief (CG).

Perinatal grief is defined as the grief that parents experience after the dead of a baby during pregnancy, birth or the first month after their birth, which includes miscarriage, ectopic pregnancy, termination, stillbirth and neo-natal deaths.

Miscarriage, generally defined as an unintended termination of the pregnancy prior to 20 weeks of gestations, is the most common type of pregnancy loss. The death of a fetus after 20 weeks gestation with a birth weight of over $500 \mathrm{~g}$ is referred to as a stillbirth.

Although grief is a natural, nonpathological phenomenon, it can lead to CG, where symptoms are more disruptive, pervasive, or long-lasting than in a normal grief response. This is especially likely if the death has occurred in a sudden, violent, or traumatic way.

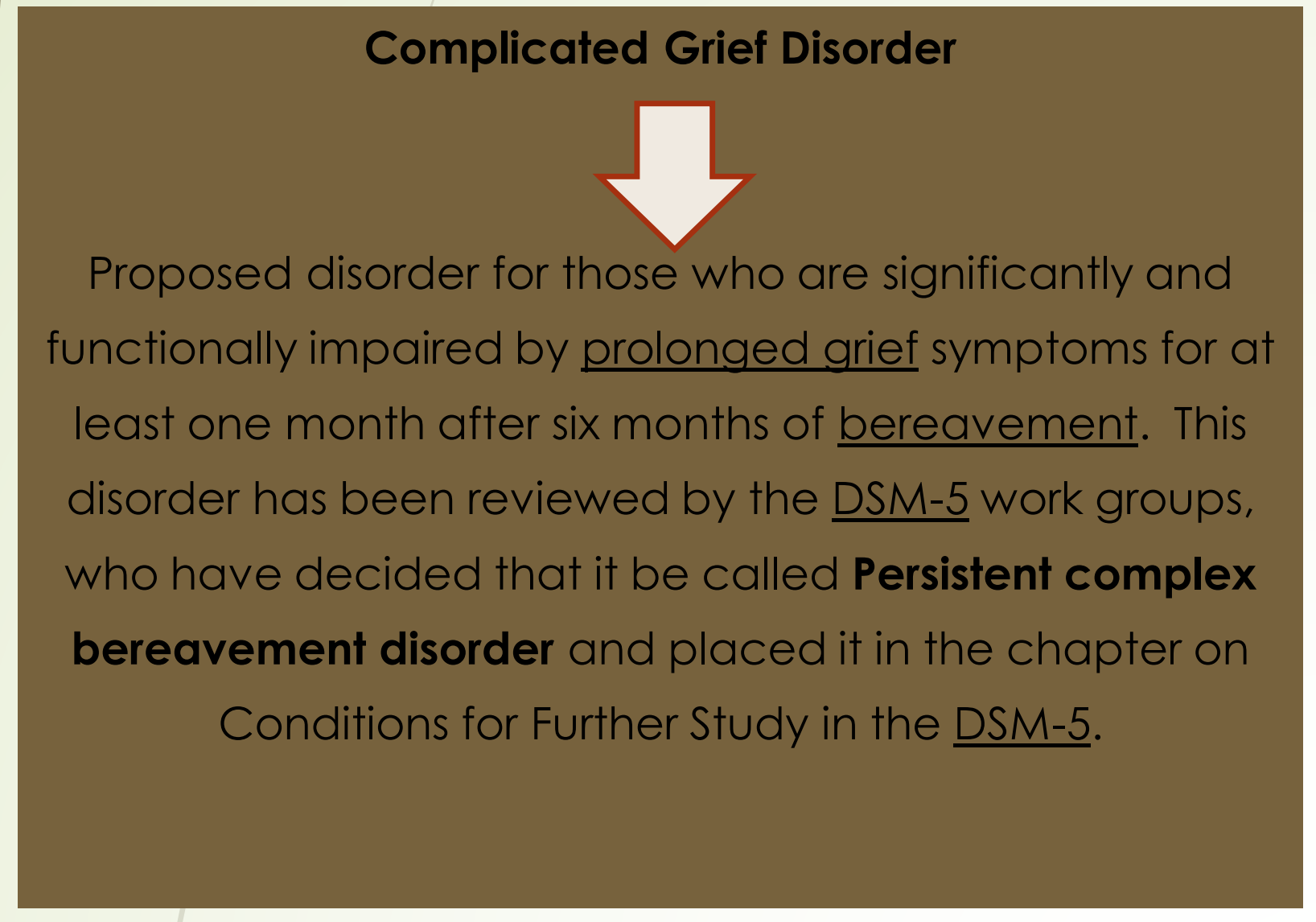

Predictors of development of complicated grief after

\section{perinatal loss include: \\ lack of social support, pre-existing relationship difficulties, or absence of surviving children, as well as ambivalent attitudes or heightened perception of the reality of the pregnancy. Risk of complicated grief was found to be especially high after termination of a pregnancy due to fetal abnormality.}

Although parents have not built up a relationship with their
infant, grief after pregnancy loss does not differ
significantly in intensity from other loss scenarios. As has
been found in bereavement involving first-degree
relatives, grief symptoms usually decrease in intensity over
the first 12 months.
Perinatal losses have also been shown to have a
substantial psychological impact on parents and families,
and are associated with post-traumatic stress, depression,
anxiety, and sleeping disorders. High levels of CG are
generally associated with a poorer state of mental health.

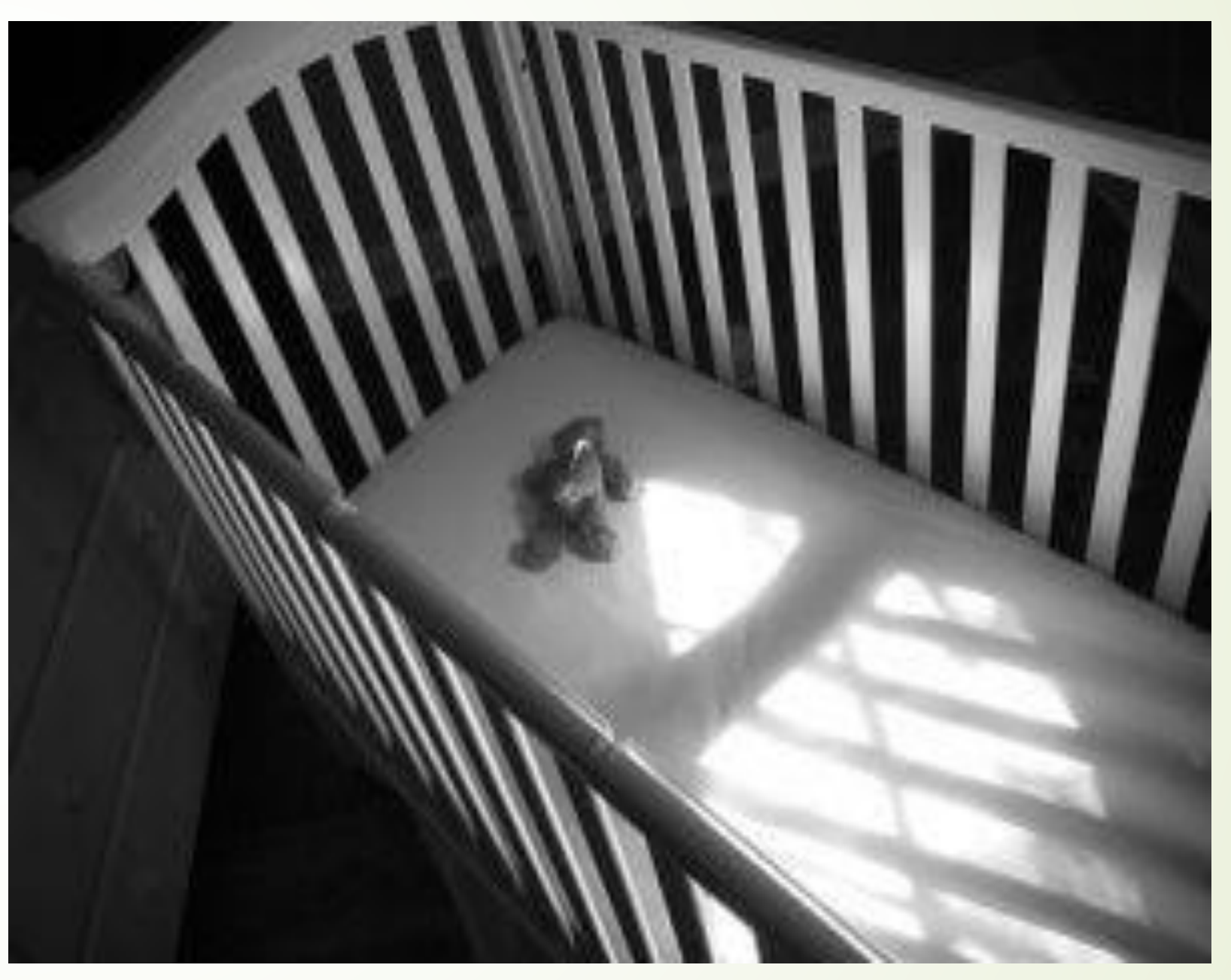

Self-blame/may prolong the normal grieving process, especially if there was a feeling of ambivalence towards the pregnancy or if the subject perceives having done something wrong (for example, smoking or jogging during pregnancy). Another unique aspect of pregnancy loss is that women feel that their bodies have failed, and that their femininity has been undermined.

An additional aggravating factor is that if the loss takes place at an early stage of pregnancy there will usually be no funeral or other rituals of mourning, and the loss may remain unacknowledged by the family and friends. Generally, the possibility of saying goodbye after the loss of a significant person is assumed to have a positive impact on the bereaved person. These issues may complicate the grieving process and increase a sense of isolation for the parents. Often parents are nowadays encouraged to hold and see their stillborn infant's dead body. There is controversy over this practice and the concept has recently been challenged by recent studies. It has been found that women who hold their deceased infant have significantly higher rates of post-traumatic stress disorder (PTSD), anxiety, and depression even 7 years after the event.

Studies have revealed that the loss of an unborn child after discovery during pregnancy of fetal malformation or severe chromosomal disorders can be considered as a traumatic life event with high psychological impact. This is especially relevant if the termination of pregnancy takes place in the $2^{\text {nd }}$ or $3^{\text {rd }}$ trimester of pregnancy.

Although it is clear that prenatal loss has a large psychological impact, there is a substantial lack of randomized controlled studies in this field of research. 